\title{
Crosscorrelation and DOA Estimation for L-Shaped Array via Decoupled Atomic Norm Minimization
}

\author{
Yu Zhang $\mathbb{D}^{1},{ }^{1}$ Yinan Sun, ${ }^{2}$ Gong Zhang $\mathbb{D}^{1},{ }^{1}$ Xinhai Wang, ${ }^{3}$ and Yu Tao ${ }^{4}$ \\ ${ }^{1}$ Key Lab of Radar Imaging and Microwave Photonics, Ministry of Education, Nanjing University of Aeronautics and Astronautics, \\ Nanjing 210016, China \\ ${ }^{2}$ Electronic Information School, Wuhan University, Wuhan 430072, China \\ ${ }^{3}$ Nanjing Marine Radar Institute, Nanjing 211153, China \\ ${ }^{4}$ School of Electronic and Information Engineering, Changshu Institute of Technology, Suzhou 215500, China \\ Correspondence should be addressed to Gong Zhang; gzhang@nuaa.edu.cn
}

Received 13 November 2020; Revised 2 December 2020; Accepted 17 March 2021; Published 30 March 2021

Academic Editor: Jun Cai

Copyright (c) $2021 \mathrm{Yu}$ Zhang et al. This is an open access article distributed under the Creative Commons Attribution License, which permits unrestricted use, distribution, and reproduction in any medium, provided the original work is properly cited.

\begin{abstract}
A novel two-phase method for two-dimensional (2D) direction-of-arrival (DOA) estimation with L-shaped array based on decoupled atomic norm minimization (DANM) is proposed in this paper. In the first phase, given the sample crosscorrelation matrix, the gridless DANM technique considering the noise and finite snapshots effects is employed to exploit the structure and sparse properties of the crosscorrelation matrix. The resulting DANM-based algorithm not only enables the crosscorrelation matrix reconstruction (CCMR) but also reconstructs the covariance matrix of the L-shaped array. Hence, sequentially, in the second phase, the conventional 2D DOA estimators for the L-shaped array can be adopted for the angle estimation. With appropriate 2D DOA estimators, the resulting proposed algorithms can not only achieve better performance but also detect more source number, compared with conventional crosscorrelation-based DOA estimators. Moreover, the proposed method, termed CCMR-DANM, not only has blind characteristic that it does not require the prior information of source numbers but also is more efficient than the existing CCMR-based counterparts. Numerical simulations demonstrate the effectiveness and outperformance of the proposed method.
\end{abstract}

\section{Introduction}

The problem of two-dimensional (2D) direction-of-arrival (DOA) estimation plays an important role in array signal processing and has attracted much interest in the area of wireless communications, radar and sonar [1-7]. For 2D DOA estimation, many array structures, such as rectangular arrays, circular arrays, and L-shaped arrays, have been developed. Among these arrays, since the L-shaped array can achieve better estimation performance than others, it has attracted a lot of attentions and many corresponding algorithms for 2D DOA estimation have been proposed in last several decades [8-15]. Moreover, these algorithms can be divided into three categories. The first is to separately estimate the angles corresponding to each uniform linear subarray based on the covariance matrix of each subarray with conventional 1D DOA estimators, such as MUSIC [16] and ESPRIT [17]. However, an extra pairing operation is needed in these algorithms [18]. The second is to jointly estimate the two angles based on the covariance matrix of the L-shaped array. They can detect more source numbers than the first ones and do not need an extra pairing $[8,9]$. The last is based on the crosscorrelation of the L-shaped array, which is naturally contaminated by less noise, compared with the covariance matrix. As a result, the corresponding algorithms can achieve a better performance in low signal-to-noise ratio (SNR) [10-15]. It is worth noting that in practical applications, all these three kinds of methods need the prior information of source numbers and are employed with sample matrices (no matter the sample covariance matrices or the 
sample crosscorrelation matrices). Note that the sample matrix is calculated from finite collected snapshots of the observed signals which is contaminated with additive noise. Hence, the sample matrix cannot equip the ideal structure of the ideal matrix, which leads to performance degradation especially in low SNR and small number of snapshots [19].

To overcome these disadvantages, the basic idea is to first reconstruct the ideal matrix from the sample matrix before employing DOA estimation methods. Inspired by this thought, several crosscorrelation matrix reconstruction(CCMR-) based methods are proposed. Specifically, crosscorrelation atomic norm minimization (CC-ANM) utilising the 2D ANM technique [20] for CCMR is proposed in [21]. And [22] grafts the covariance fitting criterion [23] for CCMR and proposes a crosscorrelation gridless sparse iterative covariance-based estimation (CC-GLS) method. However, a high-dimensional two-level Toeplitz matrix is needed to be constructed in both methods, which leads to high computational complexity [18]. In contrast, we propose an efficient decoupled ANM- (DANM-) based method for CCMR in [24], while the method does not consider the finite snapshot effect, which results in performance degradation in practical applications.

In this paper, given the sample crosscorrelation matrix, the DANM technique [18] considering the noise and finite snapshots effects is employed to exploit the structure and sparse properties of the crosscorrelation matrix. Moreover, the resulting DANM-based algorithm not only enables the crosscorrelation matrix reconstruction but also reconstructs the covariance matrix of the L-shaped array. Hence, the conventional 2D DOA estimators for the L-shaped array can be sequentially adopted for the angle estimation. The proposed two-phase method is more computationally efficient than the aforementioned two CCMR-based methods and can achieve better estimation performance compared with traditional crosscorrelation-based methods. Numerical simulations demonstrate the effectiveness and outperformance of the proposed method.

The rest of this paper is organized as follows. Section 2 presents the signal model and problem formulation for $2 \mathrm{D}$ DOA estimation with L-shaped array. Section 3 proposes an efficient DANM-based two-phase method for crosscorrelation and DOA estimation. Section 4 discusses the computational complexity and extends the proposed method to the sparse L-shaped array cases. Section 5 presents simulation results, followed by conclusions in Section 6.

Throughout this paper, $a$, a and A denote a scalar, a vector, and a matrix, respectively. $\operatorname{Tr}(\mathbf{A})$ denotes the trace of A. T(a) denotes the Hermitian Toeplitz matrix with the first column being a. $\operatorname{diag}(\mathbf{A})$ is a column vector formed from the elements of the main diagonal of $\mathbf{A}$ and diag (a) generates a diagonal matrix with the diagonal elements constructed from a. $\operatorname{vec}(\cdot)$ stacks all the columns of a matrix into a vector. $\mathbf{I}_{a}$ is an $a$-size identity matrix, and $\mathbf{I}_{\Omega}$ is a selection matrix with index set $\Omega$. $\mathbf{0}_{a}$ and $\mathbf{1}_{a}$ are the $a \times 1$ zeros and one vectors, respectively. $\otimes$ is the Kronecker product. $\mathbb{E}\{\cdot\}$ denotes expectation, and $\operatorname{Var}(\cdot)$ denotes variance. We use $(\cdot)^{T},(\cdot)^{*}$, and $(\cdot)^{H}$ to denote the transpose, the conjugate, and the conjugate transpose operation, respectively.

\section{Problem Formulation}

Consider $K$ far-field narrowband source signals $\left\{\mathbf{s}_{i}(t)\right\}_{i=1}^{K}$ impinging on an L-shaped array from distinct directions at element angles $\left\{\phi_{i}\right\}_{i=1}^{K}$ and azimuth angles $\left\{\theta_{i}\right\}_{i=1}^{K}$, as shown in Figure 1. The L-shaped array consists of two ULAs of $M$ omnidirectional sensors which are uniformly spaced with a spacing of $d$ along the $x$ axis and $y$ axis, respectively. The observed signals of the L-shaped array can be expressed as [24]

$$
\begin{aligned}
& \mathbf{x}(t)=\mathbf{A}_{x} \mathbf{s}(t)+\mathbf{n}_{x}(t), \\
& \mathbf{y}(t)=\mathbf{A}_{y} \mathbf{s}(t)+\mathbf{n}_{y}(t), t=1,2, \cdots, L,
\end{aligned}
$$

where $t$ indexes the snapshot; $L$ denotes the number of collected snapshots; and $\boldsymbol{s}(t), \mathbf{n}_{x}(t)$, and $\mathbf{n}_{y}(t)$ denote the vector of source signals and the vector of additive noise corresponding to the $x$ subarray and $y$ subarray at the snapshot $t$, respectively. $\mathbf{A}_{x}=\left[\mathbf{a}_{x}\left(\theta_{1}, \phi_{1}\right), \cdots, \mathbf{a}_{x}\left(\theta_{K}, \phi_{K}\right)\right]$ and $\mathbf{A}_{y}=\left[\mathbf{a}_{y}\left(\theta_{1}, \phi_{1}\right), \cdots, \mathbf{a}_{y}\left(\theta_{K}, \phi_{K}\right)\right]$ are the array manifold matrices of the $x$ subarray and the $y$ subarray, whose $i$ th columns are the steering vectors of the $i$ th source which satisfy

$$
\begin{aligned}
& \mathbf{a}_{x}\left(\theta_{i}, \phi_{i}\right)=\left[1, e^{-j 2 \pi 1 / 2 \cos \left(\theta_{i}\right) \sin \left(\phi_{i}\right)}, \cdots, e^{-j 2 \pi 1 / 2(M-1) \cos \left(\theta_{i}\right) \sin \left(\phi_{i}\right)}\right]^{T}, \\
& \mathbf{a}_{y}\left(\theta_{i}, \phi_{i}\right)=\left[1, e^{-j 2 \pi 1 / 2 \sin \left(\theta_{i}\right) \sin \left(\phi_{i}\right)}, \cdots, e^{-j 2 \pi 1 / 2(M-1) \sin \left(\theta_{i}\right) \sin \left(\phi_{i}\right)}\right]^{T} .
\end{aligned}
$$

Herein, the spaced distance $d$ is assumed to be equal to half of the wavelength $\lambda$. Moreover, let $f_{i, 1}=(1 / 2) \cos \left(\theta_{i}\right)$ $\sin \left(\phi_{i}\right), f_{i, 2}=(1 / 2) \sin \left(\theta_{i}\right) \sin \left(\phi_{i}\right)$ and $\Omega=\left\{\left(f_{1,1}, f_{1,2}\right)\right.$, $\left.\cdots,\left(f_{K, 1}, f_{K, 2}\right)\right\}$ denote the frequencies that correspond to the direction on $x$ and $y$ axes and the set of corresponding frequencies, respectively. Note that $\left\{f_{i, 1}, f_{i, 2}\right\} \longleftrightarrow\left\{\theta_{i}, \phi_{i}\right\}$ is the one-to-one mapping. Once the estimation of $\left\{f_{i, 1}, f_{i, 2}\right\}$ is obtained, the corresponding $\left\{\theta_{i}, \phi_{i}\right\}$ can be retrieved as

$$
\begin{aligned}
& \widehat{\phi}_{i}=\arcsin \left(2 \sqrt{f \wedge_{i, 1}^{2}+f \wedge_{i, 2}^{2}}\right), \\
& \widehat{\theta}_{i}=\arccos \left(\frac{\widehat{f}_{i, 2}}{\widehat{f}_{i, 1}}\right) .
\end{aligned}
$$

Hence, in the following paper, we consider estimation of $\left\{f_{i, 1}, f_{i, 2}\right\}$ instead of $\left\{\theta_{i}, \phi_{i}\right\}$ for notational simplicity. Moreover, in this paper, the source signals $\mathbf{s}(t)$ are assumed uncorrelated with each other and the noise $\mathbf{n}_{x}(t)$ and $\mathbf{n}_{y}(t)$ are i.i.d. additive white Gaussian random processes satisfying $\mathcal{N}\left(\mathbf{0}, \sigma^{2} \mathbf{I}_{M}\right)$ and are statistically independent of $\mathbf{s}(t)$. Therefore, under the above assumptions, we have the 


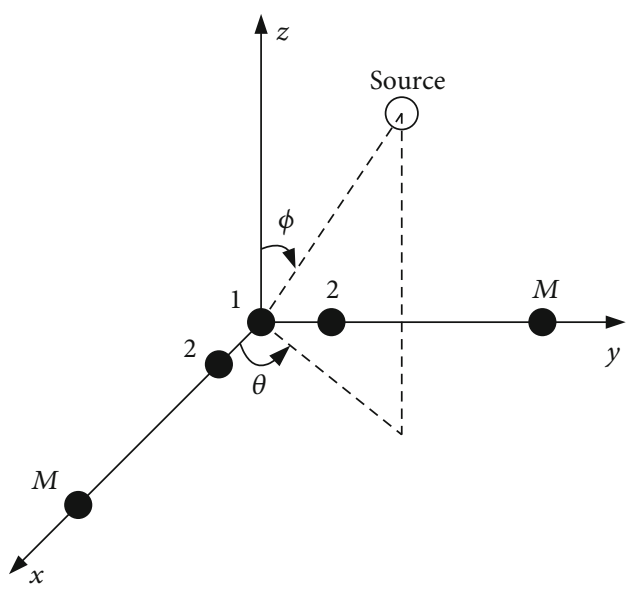

FiguRE 1: L-shaped array configuration.

crosscorrelation matrix between $\mathbf{x}(t)$ and $\mathbf{y}(t)$ can be expressed as

$$
\begin{aligned}
\mathbf{R}_{x y} & =E\left[\mathbf{x}(t) \mathbf{y}^{H}(t)\right]=\mathbf{A}_{x} \mathbf{R}_{s} \mathbf{A}_{y}+\mathbf{N}_{1}=\mathbf{R}_{c}+\mathbf{N}_{1} \\
& =\sum_{i=1}^{K} r_{i} \mathbf{a}_{x}\left(f_{i, 1}\right) \mathbf{a}_{y}^{H}\left(f_{i, 2}\right)+\mathbf{N}_{1},
\end{aligned}
$$

where $\mathbf{N}_{1}$ is the matrix with only the first element of main diagonal being $\sigma^{2}$ and zero otherwise. $\mathbf{R}_{s}=\operatorname{diag}\{\mathbf{r}\}$ is the source correlation matrix with $\mathbf{r}=\left[r_{1}, \cdots, r_{K}\right]^{T} \geq 0$, i.e., $\mathbb{E}\left(\left|s_{i}(t)\right|^{2}\right)=r_{i} . \quad \mathbf{R}_{c}=\mathbf{A}_{x} \mathbf{R}_{s} \mathbf{A}_{y}$ is the noise-free crosscorrelation matrix, while, in practical applications, $\mathbf{R}_{x y}$ can only be estimated from the finite $L$ snapshots by $\widehat{\mathbf{R}}_{x y}=1 / L \sum_{t=1}^{L} \mathbf{x}(t) \mathbf{y}^{H}(t)$, which not only is contaminated by the noise but also contains errors caused by the finite snapshot effect. The goal of this paper is to recover the noise-free crosscorrelation matrix $\mathbf{R}_{c}$ and then the unknown 2D DOAs $\left\{\theta_{i}, \phi_{i}\right\}_{i}$ from the sample crosscorrelation matrix $\widehat{\mathbf{R}}_{x y}$.

\section{Proposed Method}

In this section, we propose an efficient DANM-based crosscorrelation and DOA estimation method for L-shaped array. To this end, the standard DANM technique is firstly presented. Then, the sparse representation of the noise-free crosscorrelation matrix is presented, which enables the DANM technique to exploit its sparse property. Further, simultaneously considering the noise and finite snapshot effects, and the structure property of the crosscorrelation matrix, we propose the original DANM-based formulation for CCMR which is intractable since the structure constraint. To make the formulation tractable, an effective relaxation is proposed. Moreover, an estimation error constraint leading to easy setting of the user-specific parameter is also proposed.

3.1. Prior Art: DANM Technique. We now review the standard DANM technique for harmonic retrieval. Define a matrix $\mathbf{Z}$ as

$$
\mathbf{Z}=\sum_{k=1}^{K} \alpha_{k} \mathbf{a}_{x}\left(f_{k, 1}\right) \mathbf{a}_{y}^{H}\left(f_{k, 2}\right)
$$

where $\alpha_{k} \in \mathbb{C}$. Then, based on (5), a matrix-form atom set of infinite size is defined as $[18,15]$

$$
\mathscr{A}=\left\{\mathbf{a}_{x}\left(f_{1}\right) \mathbf{a}_{y}^{H}\left(f_{2}\right) \mid f_{1}, f_{2} \in\left[-\frac{1}{2}, \frac{1}{2}\right)\right\} .
$$

Accordingly, the atomic norm of $\mathbf{Z}$ over the atom set $\mathscr{A}$ is defined as

$$
\begin{aligned}
\|\mathbf{Z}\|_{\mathscr{A}}= & \inf \left\{\sum_{k}\left|\alpha_{k}\right| \mid \sum_{k} \alpha_{k} \mathbf{a}_{x}\left(f_{k, 1}\right) \mathbf{a}_{y}^{H}\left(f_{k, 2}\right), \mathbf{a}_{x}\left(f_{k, 1}\right) \mathbf{a}_{y}^{H}\right. \\
& \left.\cdot\left(f_{k, 2}\right) \in \mathscr{A} ; \alpha_{k} \in \mathbb{C}\right\},
\end{aligned}
$$

which seeks the sparsest (under $l_{1}$-norm measure) decomposition of $\mathbf{Z}$ over $\mathscr{A}$. Consider the matrix $\mathbf{Z}$ is contaminated by the noise matrix $\mathbf{N}$ and the matrix at hand is $\widehat{\mathbf{Z}}=\mathbf{Z}+\mathbf{N}$. Then, according to the DANM theory $[18,25]$, the atomic decomposition yields the true structure in (5), through the following DANM formulation:

$$
\tilde{\mathbf{Z}}=\arg \min _{\mathbf{Z}}\|\mathbf{Z}\|_{\mathscr{A}} \text { s.t. }\|\widehat{\mathbf{Z}}-\mathbf{Z}\|_{F}^{2} \leq \eta
$$

where $\eta$ is a user-specified parameter for error tolerance. Moreover, (8) is equivalent to the following semidefinite positive (SDP) formulation

$$
\begin{aligned}
\left\{\tilde{\mathbf{z}}_{1}, \tilde{\mathbf{z}}_{2}, \tilde{\mathbf{Z}}\right\}= & \arg \min _{z_{1}, z_{2}, \mathbf{Z}} \frac{1}{2 M}\left(\operatorname{Tr}\left(\mathrm{T}\left(\mathbf{z}_{1}\right)\right)+\operatorname{Tr}\left(\mathrm{T}\left(\mathbf{z}_{2}\right)\right)\right) \\
\text { s.t. } \quad & \|\widehat{\mathbf{Z}}-\mathbf{Z}\|_{F}^{2} \leq \eta \\
& {\left[\begin{array}{cc}
\mathrm{T}\left(\mathbf{z}_{1}\right) & \mathbf{Z} \\
\mathbf{Z}^{H} & \mathrm{~T}\left(\mathbf{z}_{2}\right)
\end{array}\right] \geqslant \mathbf{0} . }
\end{aligned}
$$

3.2. Standard DANM-Based Formulation for CCMR. Note that $\mathbf{R}_{c}=\sum_{i=1}^{K} r_{i} \mathbf{a}_{x}\left(f_{i, 1}\right) \mathbf{a}_{y}^{H}\left(f_{i, 2}\right)$. Apparently, $\mathbf{R}_{c}$ has a sparse linear atomic representation over the matrix-form atom set in (6). And we introduce a new matrix-form atomic norm

$$
\begin{aligned}
\left\|\mathbf{R}_{c}\right\|_{\mathscr{A}}^{+}= & \inf \left\{\sum_{i} r_{i} \mid \sum_{i} r_{i} \mathbf{a}_{x}\left(f_{i, 1}\right) \mathbf{a}_{y}^{H}\left(f_{i, 2}\right), \mathbf{a}_{x}\left(f_{i, 1}\right) \mathbf{a}_{y}^{H}\right. \\
& \left.\cdot\left(f_{i, 2}\right) \in \mathscr{A} ; r_{i} \geq 0, \forall i\right\} .
\end{aligned}
$$

Note that this norm differs from the original atomic norm of the standard DANM in (7), because of the extra constraint $\mathbf{r} \geq \mathbf{0}$. 
Given $\mathbf{R}_{c}$, it is possible to retrieve the components $\left\{r_{i}\right.$, $\left.f_{i, 1}, f_{i, 2}\right\}$ of its sparest representation by calculating its atomic norm. Considering the obtained at hand is the sample crosscorrelation containing the estimation error, it boils down to

$$
\begin{aligned}
& \tilde{\mathbf{R}}_{c}=\arg \min _{\mathbf{R}_{c}}\left\|\mathbf{R}_{c}\right\|_{\mathscr{A}}^{+} \\
& \text {s.t. } \mathscr{F}\left\{\widehat{\mathbf{R}}_{x y}, \mathbf{R}_{c}\right\} \leq \beta .
\end{aligned}
$$

where $\mathscr{F}\left\{\widehat{\mathbf{R}}_{x y}, \mathbf{R}_{c}\right\}$ is the function quantifying the estimation error and $\beta$ indicates the error tolerance threshold. It is similar to the DANM formulation introduced in (8), but defined on the norm $\|\cdot\|_{\mathscr{A}}^{+}$instead of $\|\cdot\|_{\mathscr{A}}$. To show the intricacy of this difference, we rewrite (11) in the following equivalent form:

$$
\begin{aligned}
\left\{\tilde{\mathbf{R}}_{c}, \tilde{\mathbf{r}}\right\} & =\min _{\mathbf{R}_{c}, \mathbf{r}}\left\|\mathbf{R}_{c}\right\|_{\mathscr{A}}=\|\mathbf{r}\|_{1} \\
\text { s.t. } \quad & \mathscr{F}\left\{\widehat{\mathbf{R}}_{x y}, \mathbf{R}_{c}\right\} \leq \beta \\
\mathbf{R}_{c} & =\mathbf{A}_{x} \operatorname{diag}(\mathbf{r}) \mathbf{A}_{y}^{H} \\
\mathbf{r} & \geq \mathbf{0} .
\end{aligned}
$$

Note that (12c) is implicit in the objective function (11) but becomes an explicit constraint because of the new nonnegative constraint (12d). Without (12d), the SDP implementation of the DANM in (9) can be used to reformulate (12) into a convex problem. However, because of the extra constraint on $\mathbf{r}$ in (12d), this problem becomes intractable, because $\mathbf{r}$ is intertwined with the other variable $\mathbf{R}_{c}$ in the form of (12c).

3.3. Effective Relaxation. To solve (12) in a tractable manner, we seek to relax $\mathbf{r} \geq \mathbf{0}$ to an effective form with respect to $\mathbf{R}_{c}$. To this end, we note that

$$
\begin{aligned}
\mathbf{R}_{c}(:, 1) & =\sum_{i=1}^{K} r_{i} \mathbf{a}_{x}\left(f_{i, 1}\right), \\
\mathbf{R}_{c}(1,:)^{H} & =\sum_{i=1}^{K} r_{i} \mathbf{a}_{y}\left(f_{i, 2}\right),
\end{aligned}
$$

where $\mathbf{R}_{c}(:, 1)$ and $\mathbf{R}_{c}(1,:)$ denote the first column and the first row of $\mathbf{R}_{c}$, respectively. Moreover, according to the property of Toeplitz matrices [26], if $\mathbf{r} \geq 0$, we have

$$
\begin{gathered}
\mathrm{T}\left(\mathbf{R}_{c}(:, 1)\right) \geqslant \mathbf{0}, \\
\mathrm{T}\left(\mathbf{R}_{c}(1,:)^{H}\right) \geqslant \mathbf{0} .
\end{gathered}
$$

Adopting (14) to replace (12d) and reformulating (12a)-(12c) into the original decoupled SDP form in (9), we reach the following effective SDP relaxation for (11):

$$
\begin{aligned}
\left\{\tilde{\mathbf{u}}_{1}, \tilde{\mathbf{u}}_{2}, \tilde{\mathbf{R}}_{c}\right\}= & \arg \min _{\mathbf{u}_{1}, \mathbf{u}_{2}, \mathbf{R}_{c}} \frac{1}{2 M}\left(\operatorname{Tr}\left(\mathrm{T}\left(\mathbf{u}_{1}\right)\right)+\operatorname{Tr}\left(\mathrm{T}\left(\mathbf{u}_{2}\right)\right)\right) \\
\text { s.t. } \quad & \mathscr{F}\left\{\widehat{\boldsymbol{R}}_{x y}, \mathbf{R}_{c}\right\} \leq \beta \\
& \mathrm{T}\left(\mathbf{R}_{c}(:, 1)\right) \geqslant \mathbf{0}, \mathrm{T}\left(\mathbf{R}_{c}(1,:)^{H}\right) \geqslant \mathbf{0} \\
& {\left[\begin{array}{cc}
\mathrm{T}\left(\mathbf{u}_{1}\right) & \mathbf{R}_{c} \\
\mathbf{R}_{c}{ }^{H} & \mathrm{~T}\left(\mathbf{u}_{2}\right)
\end{array}\right] \geqslant \mathbf{0} . }
\end{aligned}
$$

With an appropriate definition of $\mathscr{F}\left\{\widehat{\mathbf{R}}_{x y}, \mathbf{R}_{c}\right\}$ and $\beta,(18)$ can be solved successfully via off-the-shelf convex solvers, such as CVX [27].

3.4. Estimation Error Constraint. To define a nice $\mathscr{F}\left\{\widehat{\mathbf{R}}_{x y}, \mathbf{R}_{c}\right\}$ which leads to easy setting of $\beta$, we denote the estimation error matrix as

$$
\mathbf{E}=\widehat{\mathbf{R}}_{x y}-\mathbf{R}_{c} .
$$

Denote by $\varepsilon_{p, q}$ the $(p, q)$ th element of the estimation error matrix $\mathbf{E}$; then, one has the following proposition:

Proposition 1. For adequately large $L$, and $\forall p+q-2>0$, $\varepsilon_{p, q}$ is approximately circular complex Gaussian distributed with zero mean, and the variance is

$$
\begin{gathered}
\operatorname{Var}\left(\varepsilon_{p, q}\right)=\frac{1}{L}\left\{\left(\sum_{i=1}^{K} r_{i}+\sigma^{2}\right)^{2}-\sum_{i=1}^{K} r_{i}^{2}\right\} . \\
p+q-2>0, \quad 1 \leq p, q \leq M .
\end{gathered}
$$

Proof. Note that $\widehat{\mathbf{R}}_{x y}$ is estimated from the $L$ collected snapshots. Denote $\widehat{R}_{p, q}$ as the $(p, q)$ th element of $\widehat{\mathbf{R}}_{x y} \cdot x_{p}(t)$, $y_{q}(t), s_{i}(t), n_{x(p)}(t)$, and $n_{y(q)}(t)$ are similarly defined. We have $\forall p+q-2>0$,

$$
\begin{aligned}
\widehat{R}_{p, q}= & \frac{1}{L} \sum_{t=1}^{L} x_{p}(t) y_{q}^{*}(t)=\frac{1}{L} \sum_{t=1}^{L}\left(\sum_{i=1}^{K} s_{i}(t) e^{-j 2 \pi(p-1) f_{j, 1}}+n_{x(p)}(t)\right) \\
& \cdot\left(\sum_{j=1}^{K} s_{j}^{*}(t) e^{j 2 \pi(q-1) f_{j, 2}}+n_{y(q)}^{*}(t)\right) \\
= & \sum_{k=1}^{K}\left(\frac{1}{L} \sum_{t=1}^{L}\left|s_{k}(t)\right|^{2}\right) e^{-j 2 \pi(p-1) f_{k, 1}} e^{j 2 \pi(q-1) f_{k, 2}} \\
& +\frac{1}{L} \sum_{t=1}^{L} \sum_{i=1}^{K} \sum_{j=1}^{K} s_{i}(t) s_{j}^{*}(t) e^{-j 2 \pi(p-1) f_{i, 1} e^{j 2 \pi(q-1) f_{j, 2}}} \\
& +\frac{1}{L}\left[\sum_{t=1}^{L}\left(\sum_{i=1}^{K} s_{i}(t) n_{y(q)}^{*}(t) e^{-j 2 \pi(p-1) f_{i, 1}}+\sum_{j=1}^{K} s_{j}^{*}(t) n_{x(p)}(t) e^{j 2 \pi(q-1) f_{j, 2}}\right)\right] \\
& +\frac{1}{L} \sum_{t=1}^{L} n_{x(p)}(t) n_{y(q)}^{*}(t)=\widehat{\mathbb{R}}_{p, q}^{0}+\widehat{\mathbb{R}}_{p, q}^{1}+\widehat{\mathbb{R}}_{p, q}^{2}+\widehat{\mathbb{R}}_{p, q}^{3}
\end{aligned}
$$


where $\widehat{\mathbf{R}}_{p, q}^{0}, \widehat{\mathbf{R}}_{p, q}^{1}, \widehat{\mathbf{R}}_{p, q}^{2}$, and $\widehat{\mathbf{R}}_{p, q}^{3}$ denote the first, the second, the third, and the fourth summand. Note that $\mathbb{E}\left(\widehat{\mathbf{R}}_{p, q}^{0}\right)=\sum_{i=1}^{K} r_{i}$ $e^{-j 2 \pi(p-1) f_{i, 1}} e^{j 2 \pi(q-1) f_{i, 2}}=\mathbf{R}_{c}(p, q)$, where $\mathbf{R}_{c}(p, q)$ is the $(p, q)$ th element of $\mathbf{R}_{c}$. Hence, the $(p, q)$ th estimation error $\varepsilon_{p, q}$ can be appropriately estimated as $\varepsilon_{p, q}=\widehat{\mathbf{R}}_{p, q}^{1}+\widehat{\mathbf{R}}_{p, q}^{2}+\widehat{\mathbf{R}}_{p, q}^{z}$. Since $L$ is sufficiently large, $\varepsilon_{p, q}$ is approximately circular complex Gaussian distributed according to the central limit theorem [28]. Moreover, as the incident signals and the additive noise are mutually independent, we can easily obtain that the expectation of $\varepsilon_{p, q}$ is

$$
\mathbb{E}\left(\varepsilon_{p, q}\right)=\mathbb{E}\left(\widehat{\mathbf{R}}_{p, q}^{1}\right)+\mathbb{E}\left(\widehat{\mathbf{R}}_{p, q}^{2}\right)+\mathbb{E}\left(\widehat{\mathbf{R}}_{p, q}^{3}\right)=\mathbf{0},
$$

and the variance of $\varepsilon_{p, q}$ is

$$
\operatorname{Var}\left(\varepsilon_{p, q}\right)=\mathbb{E}\left(\left|\mathbf{R} \wedge_{p, q}^{1}\right|^{2}\right)+\mathbb{E}\left(\left|\mathbf{R} \wedge_{p, q}^{2}\right|^{2}\right)+\mathbb{E}\left(\left|\mathbf{R} \wedge_{p, q}^{3}\right|^{2}\right),
$$

where

$$
\begin{aligned}
& \mathbb{E}\left(\left|\mathbf{R} \wedge_{p, q}^{1}\right|^{2}\right)=\frac{1}{L} \sum_{i=1}^{K} \sum_{\substack{j=1 \\
j \neq i}}^{K} r_{i} r_{j}=\frac{1}{L}\left(\left(\sum_{i=1}^{K} r_{i}\right)^{2}-\sum_{i=1}^{K} r_{i}^{2}\right), \\
& \mathbb{E}\left(\left|\mathbf{R} \wedge_{p, q}^{2}\right|^{2}\right)=\frac{1}{L}\left(\sum_{i=1}^{K} r_{i} \sigma^{2}+\sum_{j=1}^{K} r_{j} \sigma^{2}\right)=\frac{2}{L} \sum_{i=1}^{K} r_{i} \sigma^{2}, \\
& \mathbb{E}\left(\left|\mathbf{R} \wedge_{p, q}^{3}\right|^{2}\right)=\frac{1}{L} \sigma^{4} .
\end{aligned}
$$

Substituting (21) into (20), we can directly have (17).

Denote $\operatorname{vec}(\mathbf{E})$ as the vectorized estimation error matrix, and note that the distribution property of $\varepsilon_{1,1}$ is not defined in Proposition 1; then, one has

$$
E\left[\|\operatorname{Jvec}(\mathbf{E})\|_{2}^{2}\right]=\sum_{p=1}^{M} \sum_{q=1, p+q-2>0}^{M} E\left(\left|\varepsilon_{p, q}\right|^{2}\right)=\left(M^{2}-1\right) \operatorname{Var}\left(\varepsilon_{2,1}\right),
$$

where $\operatorname{Var}\left(\varepsilon_{2,1}\right)$ is obtained by $(20)$ and $\mathbf{J}=\left[\mathbf{0}_{M^{2}}, \mathbf{I}_{M^{2}-1}\right]$ is the selection matrix by which $E\left(\left|\varepsilon_{1,1}\right|^{2}\right)$ is dropped from $\mathbf{E}$. Hence, according to Proposition 1 and (22), we can define the function $\mathscr{F}\left\{\widehat{\mathbf{R}}_{x y}, \mathbf{R}_{c}\right\}$ as

$$
\mathscr{F}\left\{\widehat{\mathbf{R}}_{x y}, \mathbf{R}_{c}\right\}=\left\|\operatorname{Jvec}\left(\widehat{\mathbf{R}}_{x y}-\mathbf{R}_{c}\right)\right\|_{2}^{2},
$$

and then, $\beta$ can be easily set as

$$
\beta=\mu\left\{\left(M^{2}-1\right) \operatorname{Var}\left(\varepsilon_{2,1}\right)\right\},
$$

where $\mu$ is a user-specific weighting factor permitting (24) to be held in a high probability. It is worth noting that the threshold $\beta$ is influenced by various factors, such as the variances of signals and noise, limited snapshots, and array geometry. In contrast, $\mu$ just introduces a scale to the threshold. Thus, $\mu$ has much smaller dynamic than $\beta$ and is easier to choose, which is verified in the Numerical Results. In the following, an approximation of $\operatorname{Var}\left(\varepsilon_{2,1}\right)$ is given.

Firstly, $\sum_{i=1}^{K} r_{i}+\sigma^{2}$ can be well estimated by averaging the diagonal elements of the observed covariance matrix along the $x$ and $y$ axes. It is worth noting that since the first diagonal element is common among the covariance matrices, it should be calculated only once. Hence, $\sum_{i=1}^{K} r_{i}+\sigma^{2}$ can be estimated by

$$
\frac{1}{(2 M-1)}\left\{\left[\operatorname{diag}\left(\mathbf{R} \wedge_{x}\right)+\operatorname{diag}\left(\mathbf{R} \wedge_{y}\right)\right]^{T} 1_{M}-\widehat{R}_{y}(1,1)\right\},
$$

where $\widehat{\mathbf{R}}_{x}$ and $\widehat{\mathbf{R}}_{y}$ denote the sample covariance matrix along the $x$ and $y$ axes estimated from the $L$ collected snapshots, respectively. In practical implementation, the diagonal elements of $\widehat{\mathbf{R}}_{x}$ can be calculated as $\operatorname{diag}\left(\widehat{\mathbf{R}}_{x}\right)=\operatorname{diag}\left(1 / L \sum_{t=1}^{L}\right.$ diag $\left.(\mathbf{x}(t)) \operatorname{diag}^{*}(\mathbf{x}(t))\right)$, as well as $\operatorname{diag}\left(\widehat{\mathbf{R}}_{y}\right) . \widehat{R}_{y}(1,1)$ is the $(1,1)$ th element of $\widehat{\mathbf{R}}_{y}$. Next, according to $(4)$, the $(p, q)$ th $(p+q-2>0)$ element of $\mathbf{R}_{x y}$ as $R_{p, q}$ can be expressed as $R_{p, q}=\sum_{i=1}^{K} r_{i} e^{j 2 \pi(p-1) f_{i, 1}} e^{-j 2 \pi(q-1) f_{i, 2}}$. Thus, the expectation of the squared modulus of $R_{p, q}$ is

$$
\begin{aligned}
\mathbb{E}\left\{\left|R_{p, q}\right|^{2}\right\}= & \sum_{i=1}^{K} r_{i}^{2}+\sum_{i=1}^{K} \sum_{\substack{i^{\prime}=1 \\
i^{\prime} \neq i}}^{K} r_{i} r_{i}^{\prime} \times \cos \left(2 \pi(p-1)\left(f_{i, 1}-f_{i^{\prime} 1}\right)\right. \\
& \left.+2 \pi(q-1)\left(f_{i^{\prime}, 2}-f_{i, 2}\right)\right) .
\end{aligned}
$$

The term $\cos \left(2 \pi(p-1)\left(f_{i, 1}-f_{i^{\prime} 1}\right)+2 \pi(q-1)\left(f_{i^{\prime}, 2}-\right.\right.$ $\left.\left.f_{i, 2}\right)\right)$ varies for different $p, q, i, i^{\prime}$. Moreover, the phase mod $\left(2 \pi(p-1)\left(f_{i, 1}-f_{i^{\prime} 1}\right)+2 \pi(q-1)\left(f_{i^{\prime}, 2}-f_{i, 2}\right), 2 \pi\right)$ is uniformly distributed in $[-\pi, \pi)$ with respect to uniform distributions of $f_{i, 1}$ and $f_{i, 2}$ within $[-0.5,0.5)$. Thus, the cosine term has a mean of zero. Further, if we take the average of the module of $\widehat{R}_{p, q},(p+q-2>0)$, the cosine term will be eliminated and only the first term is retained. Hence, we can approximate $\sum_{i=1}^{K} r_{i}^{2}$ with the average of the module of $\widehat{R}_{p, q},(p+q-2>0)$ as

$$
\sum_{i=1}^{K} r_{i}^{2} \approx \frac{\left\|\operatorname{Jvec}\left(\widehat{\mathbf{R}}_{x y}\right)\right\|_{2}^{2}}{\left(M^{2}-1\right)} .
$$

Finally, substituting (25) and (27) into (17), the approximation of $\operatorname{Var}\left(\varepsilon_{2,1}\right)$ is obtained, and then, $\beta$ can be determined via (24). With the obtained $\beta$ and the function $\mathscr{F}\left\{\widehat{\mathbf{R}}_{x y}, \mathbf{R}_{c}\right\}$ in (26), we have the proposed CCMR- 
based DANM (CCMR-DANM) method for L-shaped array as follows:

$$
\begin{aligned}
\left\{\tilde{\mathbf{u}}_{1}, \tilde{\mathbf{u}}_{2}, \tilde{\mathbf{R}}_{c}\right\}= & \arg \min _{\mathbf{u}_{1}, \mathbf{u}_{2}, \mathbf{R}_{c}} \frac{1}{2 M}\left(\operatorname{Tr}\left(\mathrm{T}\left(\mathbf{u}_{1}\right)\right)+\operatorname{Tr}\left(\mathrm{T}\left(\mathbf{u}_{2}\right)\right)\right) \\
\text { s.t. } & \left\|\operatorname{Jvec}\left(\widehat{\mathbf{R}}_{x y}-\mathbf{R}_{c}\right)\right\|_{2}^{2} \leq \beta \\
& \mathrm{T}\left(\mathbf{R}_{c}(:, 1)\right) \geqslant \mathbf{0}, \mathrm{T}\left(\mathbf{R}_{c}(1,:)^{H}\right) \geqslant \mathbf{0} \\
& {\left[\begin{array}{cc}
\mathrm{T}\left(\mathbf{u}_{1}\right) & \mathbf{R}_{c} \\
\mathbf{R}_{c}^{H} & \mathrm{~T}\left(\mathbf{u}_{2}\right)
\end{array}\right] \geqslant \mathbf{0} . }
\end{aligned}
$$

Compared with the SDP formulation of standard DANM in (9), beside the error tolerance constraint, an extra constraint in (14) is included in the proposed SDP formulation. It is an effectiveness relaxation of the positive constraint $\mathbf{r} \geq 0$, which is introduced by the prior information of the crosscorrelation matrix. Moreover, the importance of this extra constraint is verified in simulations.

3.5. DOA Retrieval. By solving (28), we note that besides the estimation of $\mathbf{R}_{c}$, the estimation of $\mathrm{T}\left(\mathbf{u}_{1}\right)$ and $\mathrm{T}\left(\mathbf{u}_{1}\right)$ also are obtained, which can be expressed in the form of an augmented matrix as

$$
\mathbf{R}_{\mathrm{A}}=\left[\begin{array}{cc}
\mathrm{T}\left(\mathbf{u}_{1}\right) & \mathbf{R}_{c} \\
\mathbf{R}_{c}^{H} & \mathrm{~T}\left(\mathbf{u}_{2}\right)
\end{array}\right] .
$$

Next, we develop three kinds of angle estimation methods based on $\mathrm{T}\left(\mathbf{u}_{1}\right)$ and $\mathrm{T}\left(\mathbf{u}_{2}\right), \mathbf{R}_{c}$, and $\mathbf{R}_{\mathrm{A}}$, respectively.

First, since $\mathrm{T}\left(\mathbf{u}_{1}\right)$ and $\mathrm{T}\left(\mathbf{u}_{2}\right)$ contain the unknown angle information, the Vandermonde decomposition-based methods such as MUSIC and ESPRIT [17, 29, 30] can be employed for angle estimation in each dimension. Of course, a pairing operation needs to be done to finally obtain the angle pairs [18].

Second, considering $\mathbf{R}_{c}$ as the noise-free crosscorrelation matrix, conventional 2D DOA estimation algorithms based on crosscorrelation [18-15] can be grafted with the proposed CCMR-DANM method for angle estimation. In this paper, we adopt the JSVD algorithm [10] as a representation for comparison in simulations.

Last, according to the decoupled atomic norm theory [18, 25], we have

$$
\mathbf{R}_{\mathrm{A}}=\left[\begin{array}{cc}
\mathrm{T}\left(\mathbf{u}_{1}\right) & \mathbf{R}_{c} \\
\mathbf{R}_{c}^{H} & \mathrm{~T}\left(\mathbf{u}_{2}\right)
\end{array}\right]=\left[\begin{array}{c}
\mathbf{A}_{x} \\
\mathbf{A}_{y}
\end{array}\right] \frac{1}{\sqrt{M}} \mathbf{R}_{s}\left[\begin{array}{l}
\mathbf{A}_{x} \\
\mathbf{A}_{y}
\end{array}\right]^{H}=\mathbf{H} \mathbf{R}_{s}^{\prime} \mathbf{H}^{H},
$$

where $\mathbf{H}=\left[\mathbf{A}_{x}^{T}, \mathbf{A}_{y}^{T}\right]^{T}$ and $\mathbf{R}_{s}^{\prime}=(1 / \sqrt{M}) \mathbf{R}_{s}$. Hence, the conventional covariance-based $2 \mathrm{D}$ DOA estimation algorithms, such as $[8,9]$, can be incorporated into the proposed CCMR-DANM method.
Remark 2. Note that all the matrices we obtained through (28) are low rank and their rank values are equal to the source numbers. In the three kinds of proposed angle estimation methods, either eigenvalue decomposition (EVD) or singular value decomposition (SVD) is taken on these low-rank matrices. Hence, we can determine the source numbers by counting the number of eigenvalues or singular values, which is larger than a predefined threshold, e.g., $0.05 \lambda_{\max }$, where $\lambda_{\max }$ is the maximum value of the eigenvalues. In this sense, the proposed methods can be done in a blind mode without knowing the source numbers a priori.

Remark 3. It is worth noting that the three kinds of proposed angle estimation methods have different behaviors in terms of estimation accuracy and computational complexity. The first Vandermonde decomposition-based method is computationally efficient but requires an extra pairing operation. The third $\mathbf{R}_{\mathrm{A}}$-based method is relatively computational expensive than the other two. The second crosscorrelationbased method is not only computationally efficient but also can achieve automatic pairing. Moreover, note that although the proposed method works on sample crosscorrelation matrix, the proposed method grafting with the $\mathbf{R}_{\mathrm{A}}$-based method can detect $2 M-1$ sources, which is larger than that of the conventional crosscorrelation-based DOA estimation methods.

In summary, the proposed CCMR-DANM method is widely applicable in practical implementations.

\section{Discussions}

Two related issues regarding the proposed solution in Section 3 are discussed in this section. First, the computational complexity of the proposed method is analyzed and compared with that of existing CCMR-based methods. Second, we extend the proposed method to the sparse L-shaped array cases.

4.1. Computational Complexity. Note that the CC-ANM, CCGLS, and the proposed method are all two-phase CCMRbased methods. They have common operations in sample crosscorrelation matrix construction and DOA estimation. The only difference is the technique used for CCMR. Hence, we only compare the computational complexities of the CCMR operation in these three methods. According to Vandenberghe and Boyd [31], the computational complexity for solving the SDP formulation of (28) is $\mathcal{O}\left\{\left(M^{2}+2 M\right)^{2}\right.$ $\left.(4 M)^{2.5}\right\}$. Moreover, the computational complexities of CCMR in the proposed CCMR-DANM, CC-ANM, and CC-GLS are listed in Table 1.

Further, Figure 2 presents the computational complexities of CCMR in different algorithms versus the number of antennas at each subarray. Obviously, the proposed CCMRDANM has the least computational cost compared with CC-ANM and CC-GLS when $M>4$. Further, the computational gaps between the proposed and the other two algorithms become large with the growth of the number of 
TABLE 1: The computational complexities of CCMR in different algorithms.

\begin{tabular}{lc}
\hline Algorithm & Computational complexity \\
\hline CC-ANM & $\mathcal{O}\left\{\left(3 M^{2}-2 M\right)^{2} M^{4.5}\right\}$ \\
CC-GLS & $\mathcal{O}\left\{\left(2 M^{2}-2 M\right)^{2} M^{4.5}\right\}$ \\
Proposed & $\mathcal{O}\left\{\left(M^{2}+2 M\right)^{2}(4 M)^{2.5}\right\}$ \\
\hline
\end{tabular}

antennas. Hence, the proposed method offers us a more efficient choice for CCMR, especially with large $M$.

4.2. Extension to Sparse L-Shaped Array Cases. In the sparse Lshaped array case, we consider there are two sparse linear array (SLA) arranged along the $x$ and $y$ axes. Moreover, each SLA is a subarray of a virtual ULA. In other words, the manifolds of the SLAs termed $\mathbf{A}_{x}{ }^{\prime}$ and $\mathbf{A}_{y}{ }^{\prime}$ can be expressed as [22]

$$
\begin{aligned}
& \mathbf{A}_{x^{\prime}}=\mathbf{I}_{\Omega_{x}} \mathbf{A}_{x}, \\
& \mathbf{A}_{y^{\prime}}=\mathbf{I}_{\Omega_{y}} \mathbf{A}_{y},
\end{aligned}
$$

where $\mathbf{I}_{\Omega_{x}}$ is the selection matrix for the SLA in the axis with $\Omega_{x}$ being the index set indicating the index of remaining antennas and $\mathbf{I}_{\Omega_{y}}$ is similarly defined. Hence, we have

$$
\mathbf{R}_{x^{\prime} y^{\prime}}=\mathbf{I}_{\Omega_{x}}\left(\mathbf{R}_{c}+\mathbf{N}_{1}\right) \mathbf{I}_{\Omega_{y}}^{H},
$$

and sequentially, the error estimation matrix can be defined as

$$
\mathbf{E}^{\prime}=\widehat{\mathbf{R}}_{x^{\prime} y^{\prime}}-\mathbf{I}_{\Omega_{x}} \mathbf{R}_{c} \mathbf{I}_{\Omega_{y}} .
$$

Moreover, in this paper, we consider that the two SLAs are two redundancy linear arrays [22], which means the virtual crosscorrelation as $\mathbf{R}_{x y}$ can be fully determined by $\mathbf{R}_{x^{\prime} y^{\prime}}$. Further, each element in $\mathbf{E}^{\prime}$ has similar distribution as that in $\mathbf{E}$. In other words, we can similarly defined the function $\mathscr{F}\left\{\widehat{\mathbf{R}}_{x^{\prime}} y^{\prime}\right.$, $\left.\mathbf{I}_{\Omega_{x}} \mathbf{R}_{c} \mathbf{I}_{\Omega_{y}}\right\}$ and the tolerant bound $\beta^{\prime}$ as those in (23) and (24), respectively. Then, we have the proposed CCMRDANM for sparse L-shaped array as

$$
\begin{gathered}
\left\{\tilde{\mathbf{u}}_{1}, \tilde{\mathbf{u}}_{2}, \tilde{\mathbf{R}}_{c}\right\}=\min _{\mathbf{u}_{1}, \mathbf{u}_{2}, \mathbf{R}_{c}} \frac{1}{2 M}\left(\operatorname{Tr}\left(\mathrm{T}\left(\mathbf{u}_{1}\right)\right)+\operatorname{Tr}\left(\mathrm{T}\left(\mathbf{u}_{2}\right)\right)\right) \\
\text { s.t. }\left\|\mathbf{J}^{\prime}\left(\operatorname{vec}\left(\widehat{\mathbf{R}}_{x^{\prime} y^{\prime}}\right)-\left(\mathbf{I}_{\Omega_{y}} \otimes \mathbf{I}_{\Omega_{x}}\right) \operatorname{vec}\left(\mathbf{R}_{c}\right)\right)\right\|_{2}^{2} \leq \beta^{\prime} \\
\mathrm{T}\left(\mathbf{R}_{c}(:, 1)\right) \geqslant 0, \mathrm{~T}\left(\mathbf{R}_{c}(1,:)^{H}\right) \geqslant 0 \\
{\left[\begin{array}{cc}
\mathrm{T}\left(\mathbf{u}_{1}\right) & \mathbf{R}_{c} \\
\mathbf{R}_{c}{ }^{H} & \mathrm{~T}\left(\mathbf{u}_{2}\right)
\end{array}\right] \geqslant 0,}
\end{gathered}
$$

where $\mathbf{J}^{\prime}$ is similarly defined as $\mathbf{J}$.

\section{Numerical Results}

In this section, we present numerical examples to verify the effectiveness of the proposed method and evaluate the performance of the proposed method for L-shaped array DOA estimation. Unless specifically stated, in simulations, we consider three source signals, i.e., $K=3$, with DOAs $\left(15^{\circ}, 10^{\circ}\right)$, $\left(25^{\circ}, 20^{\circ}\right)$, and $\left(35^{\circ}, 30^{\circ}\right)$, impinge onto the L-shaped array with $M=8$. The number of collected snapshots $L$ and Monte Carlo trials $N$ is set to 200 and 500, respectively. We use root mean square error (RMSE) to evaluate DOA estimation precision of the proposed method with comparison to the MUSIC algorithm for L-shaped array (replaced with MUSIC hereafter), the JSVD [10], the CESA [12], the CC-GLS [22], and the CRB [9]. We omit the CC-ANM for comparison since it has similar estimation performance as CC-GLS and it is more computationally expensive [22]. The RMSE of the azimuth and elevation angle estimations is defined as

$$
\mathrm{RMSE}=\frac{1}{K} \sum_{i=1}^{K} \sqrt{\frac{1}{N} \sum_{n=1}^{N}\left[\left(\phi \wedge_{i, n}-\phi_{i}\right)^{2}+\left(\theta \wedge_{i, n}-\theta_{i}\right)^{2}\right]},
$$

where $\widehat{\phi}_{i, n}$ and $\widehat{\theta}_{i, n}$ denote estimates of $\phi_{i}$ and $\theta_{i}$ from the $n$th trial, respectively.

First, to guarantee the proposed CCMR-DANM method works in a best status, it is important to choose an appropriate user-specific weighting factor $\mu$. The RMSEs of the proposed method with different $\mu$ versus SNR are presented in Figure 3. The curves in Figure 3 indicate that with different $\mu$, the resulting proposed methods have different estimation performance. Moreover, the proposed method equipped with $\mu=1$ has the best performance. Hence, in following simulations, we set $\mu=1$ to implement the proposed CCMRDANM method.

Next, let us evaluate the estimation performance of the proposed CCMR-DANM method compared with the aforementioned methods. Moreover, the proposed method without the constraint (14), termed proposed w/o 2, is also simulated for comparison. Figure 4 shows the RMSEs of these methods versus SNR. As shown in Figure 4, the proposed method is effective and outperforms both the conventional L-shaped DOA estimation methods and the existing gridless CCMR-based methods. Moreover, there is a nonnegligible gap between the proposed method and the proposed w/o 2 method. It indicates that the performance of the proposed method degrades greatly when the constraint in (14) is dropped, which means the constraint in (14) is indispensable for the proposed method.

To further examine the performance, we present the RMSEs of aforementioned methods versus the number of snapshots with $\mathrm{SNR}=0 \mathrm{~dB}$ in Figure 5. The result likewise indicates that the proposed method has better estimation accuracy than the conventional methods. Combining the estimation performance comparisons with the analysis in Remark 2, we can conclude that the proposed method not only has the better estimation performance compared with 


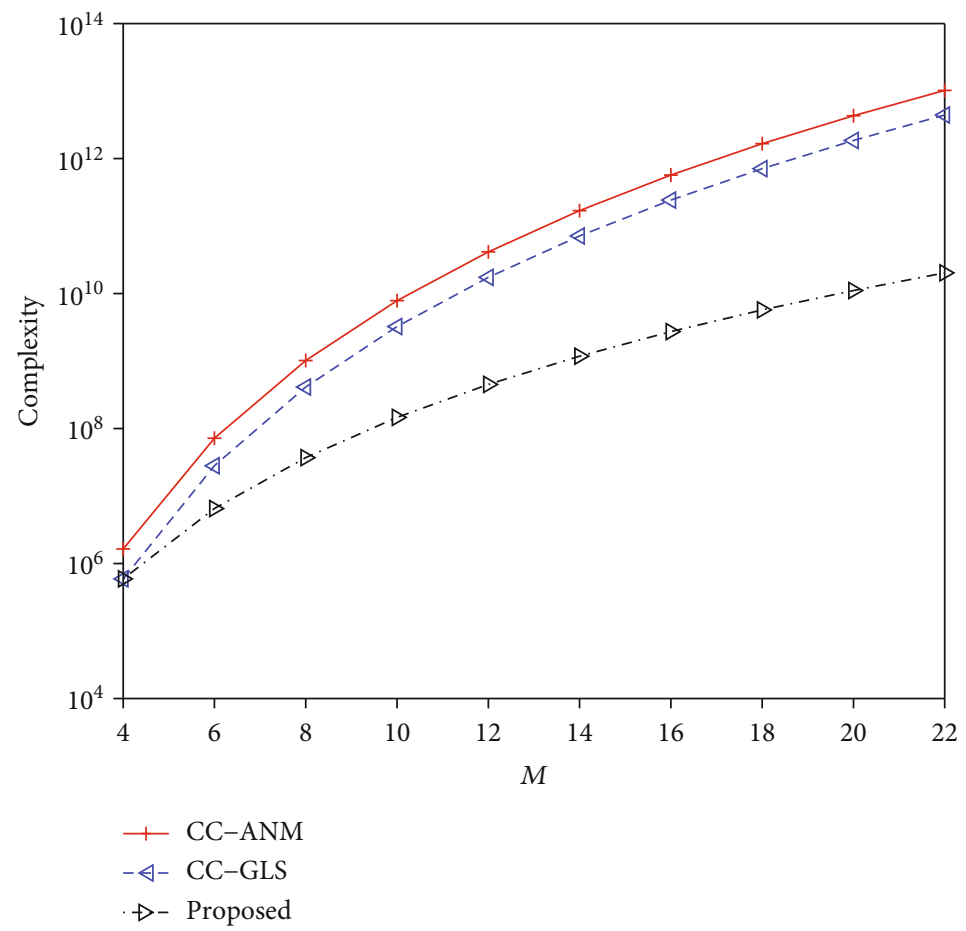

Figure 2: The computational complexities of CCMR in different algorithms versus $M$.

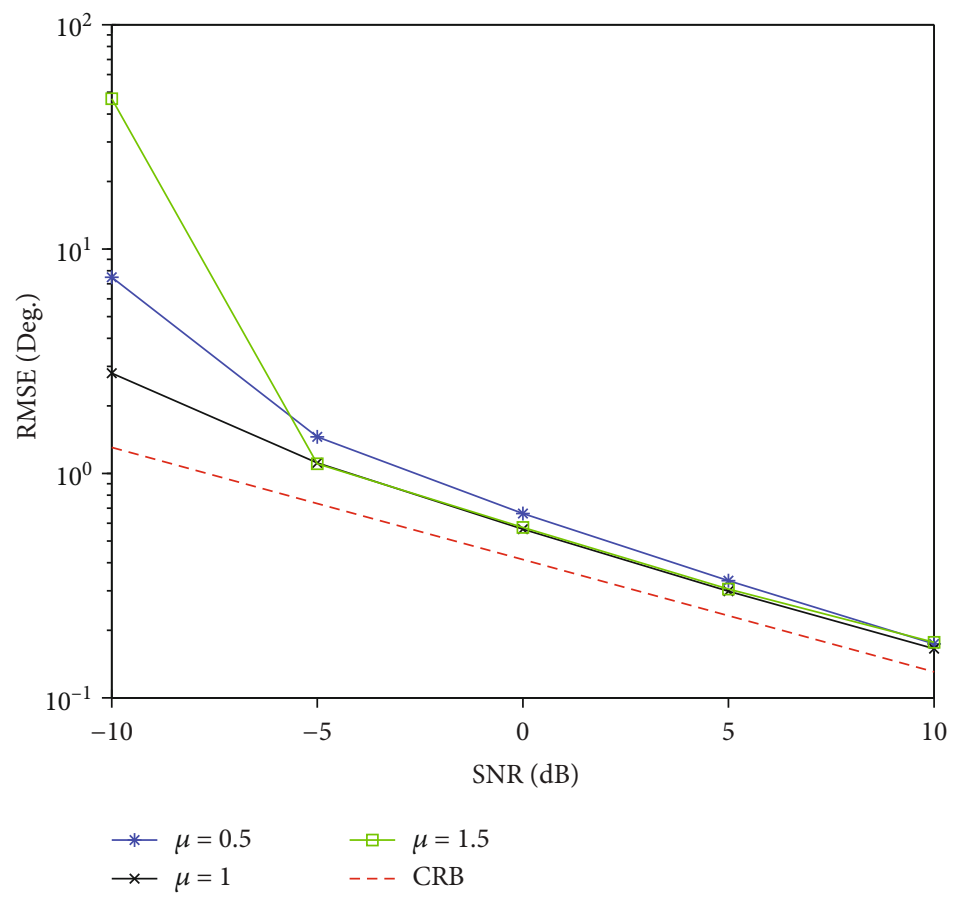

FIGURE 3: RMSE of the proposed method with different $\mu$ versus SNR.

the conventional L-shaped DOA estimation methods but also can be implemented without knowing the source numbers. Moreover, combining the estimation performance comparisons with the analysis of computational complexity in Section 4.1, we can conclude that the proposed method not only is more efficient but also has better estimation performance, compared with the existing gridless CCMR-based methods, such as CC-GLS and CC-ANM.

Last, let us consider the sparse L-shaped array cases. Assume both of the SLAs are sampled from the 8 element 


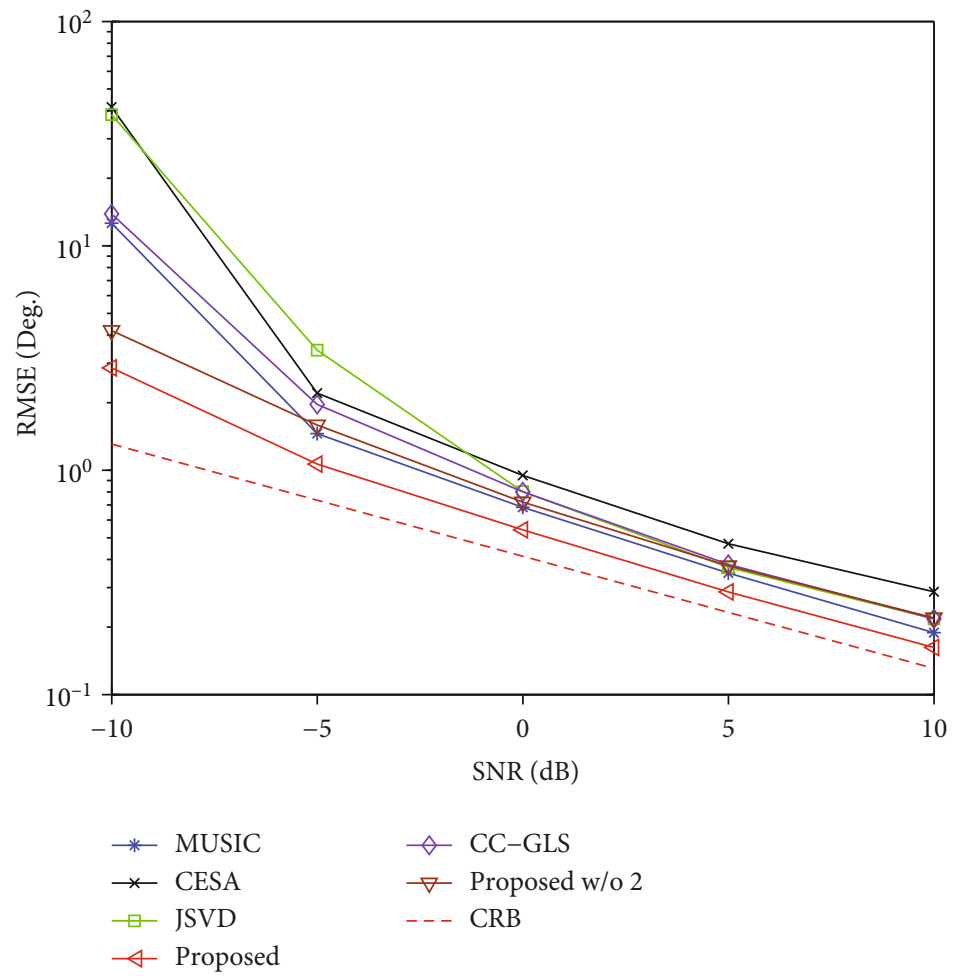

FIgURE 4: RMSE versus SNR with $L=300$.

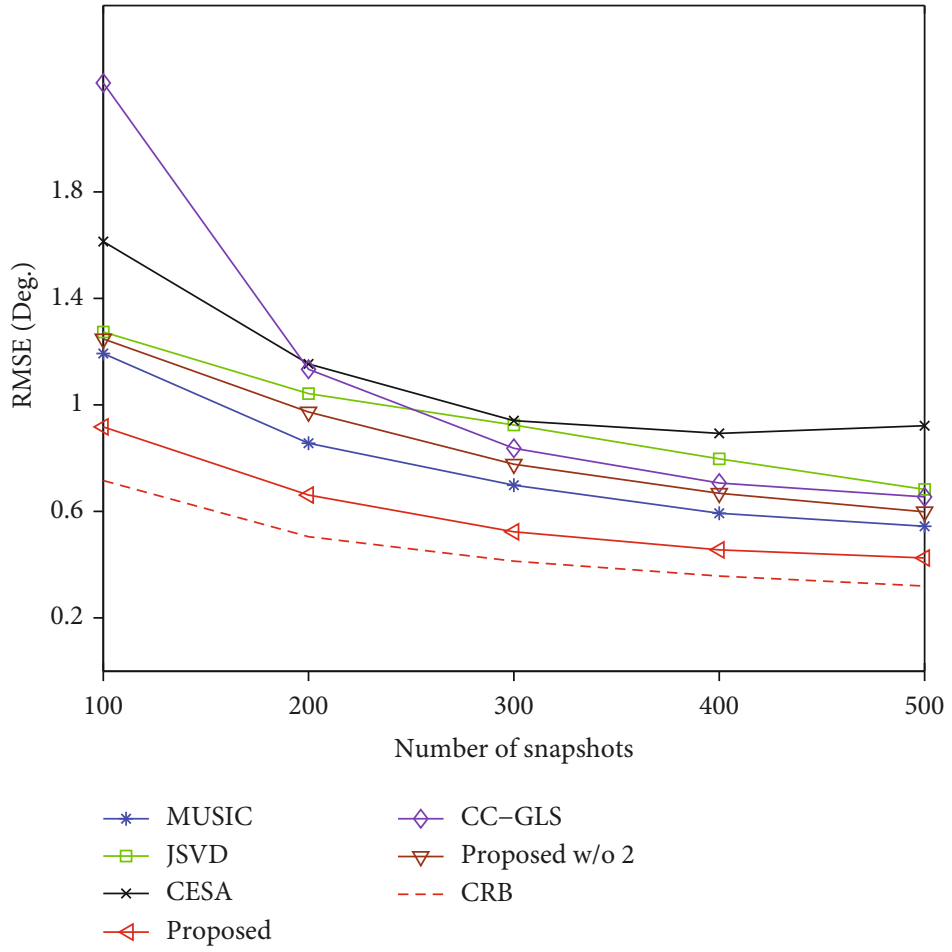

FIGURE 5: RMSE versus the number of snapshots with $\mathrm{SNR}=0 \mathrm{~dB}$.

ULA with the selection matrix $\mathbf{I}_{\Omega}$, where $\Omega=\{1,2,4,7,8\}$. And the same three source signals with DOAs $\left(15^{\circ}, 10^{\circ}\right)$, $\left(25^{\circ}, 20^{\circ}\right)$, and $\left(35^{\circ}, 30^{\circ}\right)$ impinge onto the sparse L-shaped array. As shown in Figure 6, the 2D DOAs can be clearly observed via the proposed method. In other words, the proposed method is applicable to the sparse L-shaped array cases. 


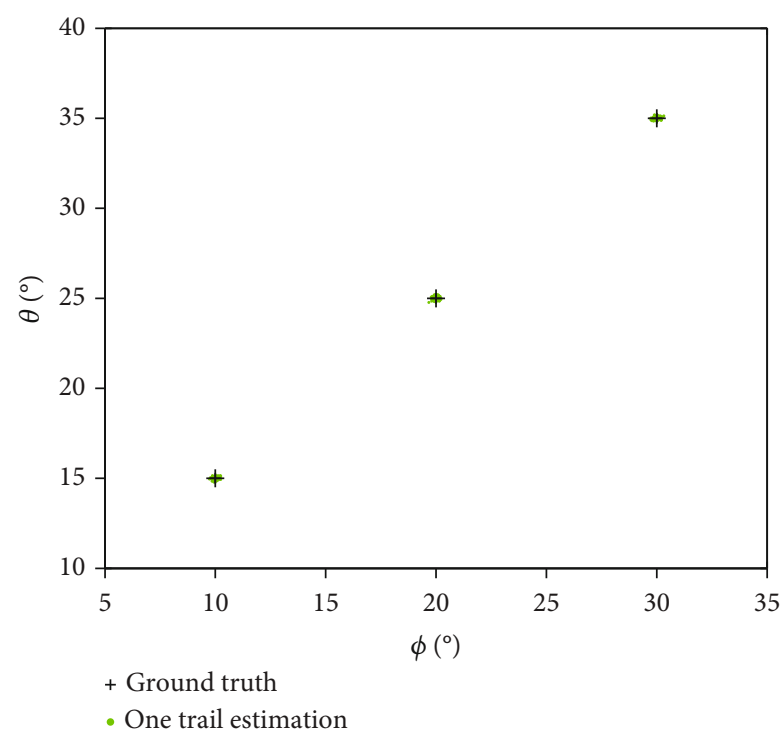

FIgURE 6: Angle estimation via the proposed CCMR-DANM in a sparse L-shaped array case with $L=200, \mathrm{SNR}=5 \mathrm{~dB}$.

\section{Conclusion}

In this paper, a new CCMR-based two-phase method for 2D DOA estimation with $\mathrm{L}$-shaped array via DANM is proposed. In the first phase, the DANM technique simultaneously considering the noise and finite snapshot effects and structure property is employed to reconstruct the noise-free crosscorrelation matrix from the sample counterpart. Then, in the second phase, conventional 2D DOA estimation methods for L-shaped array can be adopted for 2DDOA estimation. Numerical simulations demonstrate the effectiveness and outperformance of the proposed method.

\section{Data Availability}

All data sources are reliable and the corresponding author can be contacted.

\section{Conflicts of Interest}

The authors declare that they have no conflicts of interest.

\section{Acknowledgments}

This work was supported in part by the National Natural Science Foundation of China under Grant 61871218, Grant 61801211, Grant 61701046, Grant 61671241, and Grant 19KJD510004; in part by the Fundamental Research Funds for the Central Universities, China, under Grant NG2020001 and Grant 3082017NP2017421; in part by the Natural Science Foundation of Jiangsu under Grant BK20140825; in part by the Base Research Foundation under Grant NS2015040; and in part by the Funding of Key Laboratory of Radar Imaging and Microwave Photonics (Nanjing University of Aeronautics and Astronautics), Ministry of Education.

\section{References}

[1] L. Wan, X. Kong, and F. Xia, "Joint range-doppler-angle estimation for intelligent tracking of moving aerial targets," IEEE Internet of Things Journal, vol. 5, no. 3, pp. 16251636, 2018.

[2] X. Wang, L. Wang, X. Li, and G. Bi, "Nuclear norm minimization framework for DOA estimation in mimo radar," Signal Processing, vol. 135, pp. 147-152, 2017.

[3] Y.-Y. Dong and X. Chang, "Computationally efficient 2D DOA estimation for L-shaped array with unknown mutual coupling," Mathematical Problems in Engineering, vol. 2018, Article ID 5454719, 9 pages, 2018.

[4] M.-M. Liu, C.-X. Dong, Y.-Y. Dong, and G.-Q. Zhao, "Superresolution 2D DOA estimation for a rectangular array via reweighted decoupled atomic norm minimization," Mathematical Problems in Engineering, vol. 2019, Article ID 6797168, 13 pages, 2019.

[5] T. Liu, F. Wen, L. Zhang, and K. Wang, "Off-grid DOA estimation for colocated MIMO radar via reduced-complexity sparse Bayesian learning," IEEE Access, vol. 7, pp. 99907-99916, 2019.

[6] F. Wen and J. Shi, "Fast direction finding for bistatic EMVSMIMO radar without pairing," Signal Processing, vol. 173, article 107512, 2020.

[7] J. Shi, F. Wen, and T. Liu, "Nested MIMO radar: coarrays, tensor modeling and angle estimation," IEEE Transactions on Aerospace and Electronic Systems, vol. 57, no. 1, pp. 573-585, 2021.

[8] J. Li and R. T. Compton, "Two-dimensional angle and polarization estimation using the ESPRIT algorithm," IEEE Transactions on Antennas and Propagation, vol. 40, no. 5, pp. 550-555, 1992.

[9] J. Li and D. Jiang, "Joint elevation and azimuth angles estimation for L-shaped array," IEEE Antennas and Wireless Propagation Letters, vol. 16, pp. 453-456, 2017.

[10] J.-F. Gu and P. Wei, "Joint SVD of two cross-correlation matrices to achieve automatic pairing in 2-D angle estimation problems," IEEE Antennas and Wireless Propagation Letters, vol. 6, pp. 553-556, 2007.

[11] G. Wang, J. Xin, N. Zheng, and A. Sano, "Computationally efficient subspace-based method for two-dimensional direction estimation with L-shaped array," IEEE Transactions on Signal Processing, vol. 59, no. 7, pp. 3197-3212, 2011.

[12] N. Xi and L. Liping, "A computationally efficient subspace algorithm for 2-D DOA estimation with L-shaped array," IEEE Signal Processing Letters, vol. 21, no. 8, pp. 971-974, 2014.

[13] N. Tayem, K. Majeed, and A. A. Hussain, "Two-dimensional DOA estimation using crosscorrelation matrix with L-shaped array," IEEE Antennas and Wireless Propagation Letters, vol. 15, pp. 1077-1080, 2016.

[14] Y.-Y. Dong, C.-X. Dong, J. Xu, and G.-q. Zhao, "Computationally efficient 2-D DOA estimation for L-shaped array with automatic pairing," IEEE Antennas and Wireless Propagation Letters, vol. 15, pp. 1669-1672, 2016.

[15] Y.-Y. Dong, C.-x. Dong, W. Liu, H. Chen, and G.-q. Zhao, "2-D DOA estimation for L-shaped array with array aperture and snapshots extension techniques," IEEE Signal Processing Letters, vol. 24, no. 4, pp. 495-499, 2017.

[16] R. Schmidt, "Multiple emitter location and signal parameter estimation," IEEE Transactions on Antennas and Propagation, vol. 34, no. 3, pp. 276-280, 1986. 
[17] R. Roy and T. Kailath, "ESPRIT-estimation of signal parameters via rotational invariance techniques," IEEE Transactions on Acoustics, Speech, and Signal Processing, vol. 37, no. 7, pp. 984-995, 1989.

[18] Z. Zhang, Y. Wang, and Z. Tian, "Efficient two-dimensional line spectrum estimation based on decoupled atomic norm minimization," Signal Processing, vol. 163, pp. 95-106, 2019.

[19] Y. Zhang, G. Zhang, and X. Wang, "Array covariance matrixbased atomic norm minimization for off-grid coherent direction-of-arrival estimation," in 2017 IEEE International Conference on Acoustics, Speech and Signal Processing (ICASSP), pp. 3196-3200, New Orleans, LA, USA, 2017.

[20] Y. Chi and Y. Chen, "Compressive two-dimensional harmonic retrieval via atomic norm minimization," IEEE Transactions on Signal Processing, vol. 63, no. 4, pp. 1030-1042, 2015.

[21] X. Wu, W.-P. Zhu, and J. Yan, "Gridless two-dimensional DOA estimation with L-shaped array based on the crosscovariance matrix," in 2018 IEEE International Conference on Acoustics, Speech and Signal Processing (ICASSP), pp. 3256-3260, Calgary, AB, Canada, 2018.

[22] X. Wu, W.-P. Zhu, and J. Yan, "An efficient gridless 2-D DOA estimation method for sparse and uniform L-shaped arrays," in 2019 IEEE 20th International Workshop on Signal Processing Advances in Wireless Communications (SPAWC), pp. 15, Cannes, France, 2019.

[23] P. S. Hongbin Li and J. Li, "Computationally efficient maximum likelihood estimation of structured covariance matrices," IEEE Transactions on Signal Processing, vol. 47, no. 5, pp. 1314-1323, 1999.

[24] Y. Zhang, G. Zhang, and X. Wang, "Cross-correlation matrixbased atomic norm minimization for L-shaped array DOA estimation," in 2017 IEEE International Conference on Signal Processing, Communications and Computing (ICSPCC), pp. 1-4, Xiamen, China, 2017.

[25] Z. Tian, Z. Zhang, and Y. Wang, "Low-complexity optimization for two-dimensional direction-of-arrival estimation via decoupled atomic norm minimization," in 2017 IEEE International Conference on Acoustics, Speech and Signal Processing (ICASSP), pp. 3071-3075, New Orleans, LA, USA, 2017.

[26] Y. Zhang, G. Zhang, Y. Kong, and F. Wen, "Gridless sparsitybased DOA estimation for sparse linear array," The Journal of Engineering, vol. 2019, no. 20, pp. 6629-6632, 2019.

[27] M. Grant and S. Boyd, "CVX: Matlab software for disciplined convex programming, version 2.1," 2014, http://cvxr.com/cvx.

[28] D. C. Montgomery and G. C. Runger, Applied Statistics and Probability for Engineers, John Wiley \& Sons, 2007.

[29] P. Stoica and A. Nehorai, "MUSIC, maximum likelihood, and Cramer-RAO bound," IEEE Transactions on Acoustics, Speech, and Signal Processing, vol. 37, no. 5, pp. 720-741, 1989.

[30] P. Stoica and A. Nehorai, "MUSIC, maximum likelihood, and Cramer-Rao bound: further results and comparisons," IEEE Transactions on Acoustics, Speech, and Signal Processing, vol. 38, no. 12, pp. 2140-2150, 1990.

[31] L. Vandenberghe and S. Boyd, "Semidefinite programming," SIAM Review, vol. 38, no. 1, pp. 49-95, 1996. 